"C 2019 IEEE. Personal use of this material is permitted. Permission from IEEE must be obtained for all other uses, in any current or future media, including reprinting/republishing this material for advertising or promotional purposes, creating new collective works, for resale or redistribution to servers or lists, or reuse of any copyrighted component of this work in other works." 


\title{
The effects of different tracking tasks on muscle synergy through visual feedback
}

\author{
Yao Huang ${ }^{1}$, Rong Song ${ }^{2 *}$, Wenhui Chen ${ }^{1}$, Hairong $\mathrm{Yu}^{1}$, Ahmadreza Argha ${ }^{3}$, \\ Branko G. Celler ${ }^{3}$, and Steven $\mathrm{Su}^{1}$
}

\begin{abstract}
By recruiting a modular organization of muscle with relative activities, the arm motion can be indicated by the neural system and generated for performing a variety of motor tasks. In this study, a Non-negative Matrix Factorization with initial estimation is applied to identify and extract primary muscle synergies and their activation patterns from the processed EMG recordings during three multidirectional tracking tasks with visual feedback interaction. The effects of task variety and tracking accuracy by visual feedback on muscle synergies and their activation patterns are explored by statistic analysis. The results showed that only the task variety affected what synergies were indicated by the neural system, but both task variety and visual feedback affected the duration and magnitude of the primary synergies. Thus, for active rehabilitation application, it is advised that if the purpose is to enhance the synergy indication from the neural system, the task completion accuracy should be emphasized, but if the purpose is to expand the motion area, the task variety should be diversified.
\end{abstract}

\section{INTRODUCTION}

A typical hypothesis for movement generation is the existence of muscle synergy which is recruiting a set of muscles in performing activities at different relative levels by the central nervous system (CNS) [1]. The muscle synergies during a task are combined by both shared and task-specific synergies with the indication from the motor cortical areas

\footnotetext{
${ }^{1}$ Y. Huang, W. Chen, H. Yu and S. Su are with Biomedical Engineering School, Faculty of Engineering and Information Technology, University of Technology Sydney, Ultimo, NSW, Australia.

$2 * R$. Song is with the School of Biomedical Engineering, Sun Yat-Sen University, Guangzhou, P. R. China. songrong@mail.sysu. edu.cn

${ }^{3}$ A. Argha and B.G. Celler are with the School of Electrical Engineering and Telecommunications, University of New South Wales, Sydney, Australia.
}

and the afferent systems [2]. The synergy theory facilitates in both motor control and motor learning by contributing in exploration about how the motor system constructing a large set of movements.

The muscle synergies as putative modules of muscle system are typically identified and extracted from electromyographic (EMG) signals by dimensionality reduction algorithms such as Non-negative Matrix Factorization (NNMF) [3]. The purpose of any algorithms for the procedure is improving the accuracy of the modular decompositions for reconstructing the EMG data. Most of the previous researches try to explore what kinds of task-specific muscle synergies are indicated during vast tasks and contribute to motion classification and motion analysis [4], [5]. The task complexity in [4], [5] is relatively singular (e.g. one direction rectilinear motion) because of the muscle synergies need to be extracted within a period of time. Whether muscle synergies of a complex task can be extracted with high accuracy needs to be explored. For active rehabilitation, when participants complete tasks through virtual interactions, whether their efforts on improving task competition will affect the neural system in controlling movements is unclear.

For these reasons, in this study, three complex tracking tasks together with visual interaction are designed. The NNMF with an extra initial estimation is applied to extract primary muscle synergies from grouping processed EMG recordings during these tasks. The main purpose of this paper is to identify the effects on muscle synergy for these designed tasks. Both task variety (TV) and tracking accuracy (TA) by visual feedback are considered for evaluating their effects by statistic analysis. The results of this study indicate that only the TV 
affected the synergies adopted by the neural system, but both TV and TA affected the durations and magnitudes of the primary synergies.

\section{METHOD}

\section{A. Experiment design}

Three target movements with different shapes (Triangle, Square and Circle) in different dimensions are designed as shown in Fig. 1. The target movements are coding into a cursor's moving trajectories. The target cursor moves in a twodimensional surface as displayed in a screen for providing a continuous visual stimulation. The actual position of the wrist is also displayed as a cursor. Intuitively, when the participant is executing a task, he receives two different visual effects. One is the target cursor's trajectory which is defined as the Task Variety (TV), and the other one is the gap between the target and actual cursors which is defined as the tracking accuracy (TA) by visual feedback. The TV has three values corresponding to different tasks. The level of TA is processed later.

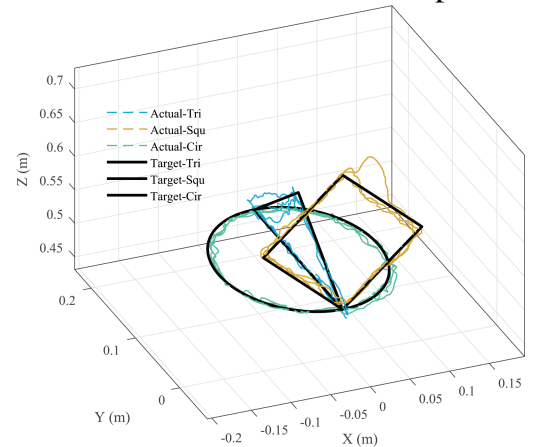

Fig. 1. The trajectories of three target tasks and actual tracking movements. Tri: Triangle, Squ: Square, Cir: Circle.

The start position of every task is the same. The length of the long side of the isosceles triangle is $0.2 \mathrm{~m}$, and the short sides are $\sqrt{0.2} \mathrm{~m}$. The length of the square sides is $\sqrt{0.2} \mathrm{~m}$. The radius of the circle is $0.1 \mathrm{~m}$. The duration time of every task is 20 seconds, and the cursor moves with constant velocity during each task. All three tasks are required to finish three times as three trails.

\section{B. Data acquisition and processing}

One healthy female is recruited and signed the written informed consent form with approvement from the Human Ethics Committee of Sun Yatsen University. The actual position of her wrist is recorded by a motion tracking system (Optitrack, USA) at $100 \mathrm{~Hz}$. The surface EMG signals of six muscles (Biceps brachii-BIC, Triceps brachii-TRI, anterior, medial and posterior part of Deltoid-DA, DM, and DP, Brachioradialis-BR) are recorded at $1000 \mathrm{~Hz}$ and amplified (gain at 5000) [6]. The raw EMG signals are bandpass filtered $(10-400 \mathrm{~Hz})$, full-wave rectified, and Butterworth low-pass filtered ( $20 \mathrm{~Hz}$ cutoff) into EMG envelops. The EMG envelops were down-sampled for synchronising.

For accurately extracting the muscle synergies, the down-sampled EMG envelops during every tracking task are separated into 40 samples. For each sample, there is a total of 50 EMG envelop data points of each muscle for 0.5 seconds. Similarly, the average (AVG), standard deviation (STD) and root mean square (RMS) values of TA in two main dimensions $\left(D_{1}, D_{2}\right)$ during each sample is calculated. For the level of TA during the whole task, the TA in $D_{1}, D_{2}$ and their square root ( $D_{12}$, i.e. the linear gap between two cursors) are grouped into 17 levels with evenly spaced. Similarly, the level of TA of all envelop samples, the AVG, STD and RMS of TA in $D_{1}, D_{2}$ are grouped.

\section{Muscle synergy extraction}

The muscle synergies during every EMG envelops sample are extracted by NNMF algorithm from the EMG envelop samples as follows [7], [8]:

$$
M_{j}(t)=\sum_{i=1}^{N} h_{i j} w_{i}(t)+\epsilon(t)
$$

where $M_{j}$ is the normalized EMG envelopes during every sample period of $j^{\text {th }}(j \in(1,6))$ muscle, $h_{i j}$ is the time-independent weight of $j^{\text {th }}$ muscle in $i^{\text {th }}$ muscle synergy, $w_{i}(t)(S T L \times n$ matrix $)$ is the timevarying basic activation patterns vector for showing the amplitude and duration time of the $i^{t h}$ synergy, $N \in(1,5)$ is the number of extracted synergy, and $\epsilon(t)$ is noise in time series.

For choosing the minimum number of muscle synergies and selecting the most precise synergies and their patterns, the root-mean-square residual $(D)$ between the original EMG envelopes and the 
reconstructed EMG envelopes $\left(M_{R e}=H \times W\right)$ is used for evaluation. The value of $D$ is required as smaller than $10^{-4}$ [7]. The Variance Accounted For (VAF) is used to evaluate how accurate the algorithm is [5], [9]. The $V A F$ is calculated as follows, and is required as larger than $99.95 \%$ :

$$
V A F=1-\sum_{i=1}^{n}\left(M-M_{R e}\right)^{2} / \sum_{i=1}^{n} M^{2}
$$

During the synergy extraction, the residual and VAF values of the repeated multiplicative NNMF algorithm with random initial estimation show high variance. Therefore, an initial estimation of synergies and their patterns are found by using the multiplicative algorithm (Iiterations $=10$, replicate $=$ $10)$. With the initial estimation, the alternating least squares algorithm can rapidly estimate synergies and their patterns with better parameters' performance without high variance [10].

The number of extracted synergies of a task is constrained by the mean values of both $D$ and $V A F$ during all samples. The synergies of different samples are compared by using person correlation coefficients (PCC) for similarity. Only those synergies with PCC values higher than $85 \%$ are regarded the same. The mean values of the same synergies are defined as the primary synergies.

\section{Statistic analysis}

The effects of the TV values and the levels of AVG, STD and RMS of TA in two main dimensions on the primary muscle synergies $(H)$ are analysed by two-way ANOVA statistic test in sample orders. Similarly, the effects of the TV values and the levels of TA in $D_{1}, D_{2}$ and $D_{12}$ on the activation patterns of primary muscle synergies $(W)$ are analysed by two-way ANOVA statistic test in time series.

\section{RESULTS AND DISCUSSION}

\section{A. NNMF with the advanced procedure}

The results of $D$ and $V A F$ (Fig. 2) showed that the advanced procedure of NNMF for extracting muscle synergy could improve the estimation accuracy. Therefore, the extraction standard can only be applied for selecting the synergy number after using the NNMF with the initial estimation.

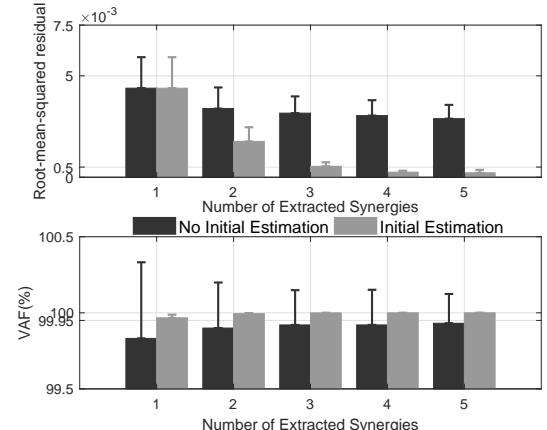

Fig. 2. The root-mean-squared residual and VAF of NNMF without or with initial estimation for extracting synergies.

\section{B. Muscle synergies and patterns}
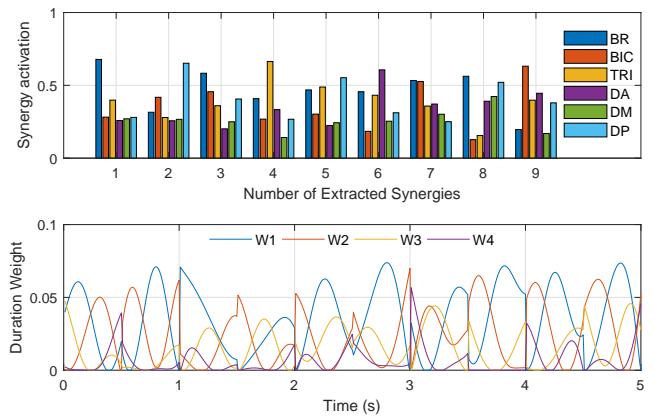

Fig. 3. Muscle synergies and basic activation patterns (W1-4).

The primary muscle synergies and their activation patterns during the first 5 seconds of a trail of triangle task is shown in Fig. 3 as an example. The number of muscle synergies was nine of all 120 samples but only four synergies were extracted in each example of this task. The first and second synergies were found in over 90 samples, and the third and fourth were found in over 50 samples. The rest synergies were found in no more than 20 samples. For the basic activation patterns, the four lines were for the primary synergies of each sample. The results can be supported by Delis et al. in task discrimination objectives by synergy difference [5].

\section{Effects on muscle synergy}

The effects of the TV and the TA by visual feedback on the primary muscle synergies and their basic activation patterns are summarised in Table I. In the Table I, the $D_{1}, D_{2}, D_{12}$ are the TA in the two main dimensions and their square root, and the 
$A V G, S T D$ and $R M S$ are the average, standard deviation and root mean square of TA.

The TV highly affected primary muscle synergies. For the combined elbow synergies, the $A V G$ and $R M S$ of TA levels in the $D_{1}$ dimension also affected what kind of synergies is indicated by the CNS system.Both the TV and TA in $D_{1}, D_{2}$ and $D_{12}$ affected the basic activation patterns of those primary muscle synergies. It indicated that no matter what primary synergies were indicated, the onset, offset and duration of the most important synergies changed with different tasks and the varying gap between actual and target positions [11].

TABLE I

EFFECTS OF TASK VARIETY (TV) AND TRACKING ACCURACY (TA) ON MUSCLE SYNERGIES (H) AND PATTERNS (W)

\begin{tabular}{|c|c|c|c|c|c|c|c|}
\hline \multirow{2}{*}{$\mathbf{H}$} & \multirow{2}{*}{ TV } & \multicolumn{3}{|c|}{$\overline{\overline{D_{1}}}$} & \multicolumn{3}{|c|}{$\overline{\overline{D_{2}}}$} \\
\hline & & AVG & STD & RMS & AVG & STD & RMS \\
\hline M1-6 & $<0.05$ & & & & & & \\
\hline$M_{E}$ & $<0.05$ & 0.05 & & $<0.05$ & & & \\
\hline$M_{S}$ & 0.05 & & & & & & \\
\hline$\overline{\mathbf{W}}$ & TV & $\overline{\overline{D_{1}}}$ & $\overline{\overline{D_{2}}}$ & $\overline{\overline{D_{12}}}$ & \multicolumn{3}{|c|}{ Effects Order } \\
\hline W1 & $<0.001$ & $<0.001$ & $<0.001$ & $<0.001$ & \multicolumn{3}{|c|}{$T V<D_{2}<D_{1}<D_{12}$} \\
\hline W2 & $<0.001$ & $<0.001$ & $<0.001$ & $<0.001$ & \multicolumn{3}{|c|}{$T V<D_{1} \approx D_{2}<D_{12}$} \\
\hline W3 & $<0.001$ & $<0.001$ & $<0.001$ & $<0.001$ & \multirow{2}{*}{\multicolumn{3}{|c|}{$T V<D_{1}<D_{2}<D_{12}$}} \\
\hline W4 $*$ & $<0.001$ & $<0.001$ & $<0.001$ & $<0.001$ & & & \\
\hline W5 * & $<0.001$ & 0.003 & $<0.001$ & $<0.001$ & \multicolumn{3}{|c|}{$T V<D_{1}<D_{12}<D_{2}$} \\
\hline
\end{tabular}

\section{CONCLUSIONS}

This paper attempts to demonstrate the effects of task variety (TV) and the tracking accuracy (TA) by visual feedback on muscle synergy and their activation patterns. The statistic analysis showed that the muscle synergies are highly related TV but their durations and activations are affected by both TV and TA. For further study, the results may suggest that during early rehabilitation for patients with neural system injury, it is essential to provide diversity tasks for evacuating different synergies to be utilized, but during later rehabilitation, the accuracy of visual tracking feedback should be advised to reach high accuracy tracking motion for acquiring synergies similar to healthy people.

\section{ACKNOWLEDGMENT}

This work was supported by the Australia-China Joint Institute for Health Technology and Inno- vation established by Sun Yat-sen University and University of Technology Sydney.

\section{REFERENCES}

[1] M. C. Tresch and A. Jarc, "The case for and against muscle synergies," Current opinion in neurobiology, vol. 19, no. 6 , pp. 601-607, 2009.

[2] E. Bizzi and V. C. Cheung, "The neural origin of muscle synergies," Frontiers in computational neuroscience, vol. 7, p. 51, 2013.

[3] M. C. Tresch, V. C. Cheung, and A. d'Avella, "Matrix factorization algorithms for the identification of muscle synergies: evaluation on simulated and experimental data sets," Journal of neurophysiology, vol. 95, no. 4, pp. 2199 2212, 2006.

[4] Y. P. Ivanenko, R. E. Poppele, and F. Lacquaniti, "Five basic muscle activation patterns account for muscle activity during human locomotion," The Journal of physiology, vol. 556, no. 1, pp. 267-282, 2004.

[5] I. Delis, S. Panzeri, T. Pozzo, and B. Berret, "Taskdiscriminative space-by-time factorization of muscle activity," Frontiers in human neuroscience, vol. 9, p. 399, 2015.

[6] Y. Huang, Q. Yang, Y. Chen, and R. Song, "Assessment of motor control during three-dimensional movements tracking with position-varying gravity compensation," Frontiers in neuroscience, vol. 11, p. 253, 2017.

[7] A. d'Avella, A. Portone, L. Fernandez, and F. Lacquaniti, "Control of fast-reaching movements by muscle synergy combinations," Journal of Neuroscience, vol. 26, no. 30, pp. 7791-7810, 2006.

[8] G. Martino, Y. P. Ivanenko, A. d'Avella, M. Serrao, A. Ranavolo, F. Draicchio, G. Cappellini, C. Casali, and F. Lacquaniti, "Neuromuscular adjustments of gait associated with unstable conditions," Journal of neurophysiology, vol. 114, no. 5, pp. 2867-2882, 2015.

[9] J. Roh, W. Z. Rymer, E. J. Perreault, S. B. Yoo, and R. F. Beer, "Alterations in upper limb muscle synergy structure in chronic stroke survivors," Journal of neurophysiology, vol. 109, no. 3, pp. 768-781, 2012.

[10] M. W. Berry, M. Browne, A. N. Langville, V. P. Pauca, and R. J. Plemmons, "Algorithms and applications for approximate nonnegative matrix factorization," Computational statistics \& data analysis, vol. 52, no. 1, pp. 155173, 2007.

[11] K. Nazarpour, A. Barnard, and A. Jackson, "Flexible cortical control of task-specific muscle synergies," Journal of Neuroscience, vol. 32, no. 36, pp. 12 349-12 360, 2012. 